\title{
Nutritional, Bone, and Physical Fitness States of Jockeys in Chronic Repetition of Short-Term Weight Loss.
}

\author{
Jeon $\mathrm{S}^{1}$, Cho $\mathrm{K}^{1}$, Ok G ${ }^{1,2}$, and Park $\mathrm{H}^{1}$
}

${ }^{1}$ Exercise Biochemistry Lab; Physical Education; Kyung Hee University; Korea. ${ }^{2}$ Counselling, Health, and Kinesiology; Texas A\&M University-San Antonio; San

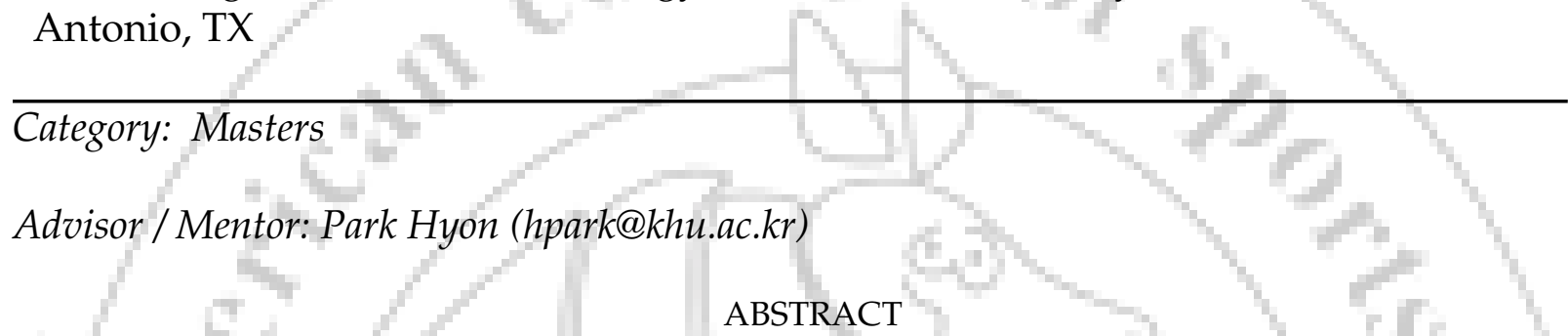

Professional jockeys are required to fit their weight, mainly weight loss, for two game days every weekend during racing season which usually lasts 10 months a year in Korea. The repetitive routine weight loss protocol is performed for 4 to 5 days a week include two game days. This situation would have brought some negative effects on the professional jockeys' overall health and physical condition with the relation of their performance. The purpose of the present study was: 1) to examine the weight loss habits of projockeys, and 2) effects of chronic short-term weight loss on nutritional status, bone health, and physical fitness of them. Pro jockeys $(n=43)$ completed a questionnaire for the assessment of weight loss habits. Pro jockeys ( $n=10$, around 12 years of career) were compared with trainee jockeys $(n=10$, less than 1 years of career) for their nutritional status, bone health, and physical fitness. The nutritional status was measured by a computerized program (CAN Pro 2.0). Bone mineral density was measured by DEXA (Hologic, QDR4500W, USA). All participants performed grip strength test, standing on one leg with eyes closed test, and side step test for the assessment of physical fitness. According to the result, more than $80 \%$ of pro jockeys underwent a routine weight loss process every week, for around $1 \mathrm{~kg}$, within 2 to 3 days prior to the game days by diet mainly and/or exercise/sauna. Around $70 \%$ of them reported severe fatigue during the weight loss period. There were no significant differences in nutritional status between two groups. However, they consume average of $1,100 \mathrm{Kcal}$ a day reflecting 21 to $80 \%$ of recommended daily consumption during weight loss periods. In bone health, six trainee jockeys and two pro jockeys were diagnosed as osteopenia. One pro jockey was diagnosed as osteoporosis. No significant difference was found in fitness factors between two groups. In conclusion, chronic exposure to short-term weight loss may result in serious health problems especially on bone health. For the jockeys in Korea, balanced diet program and the related education on weight management are strongly required..

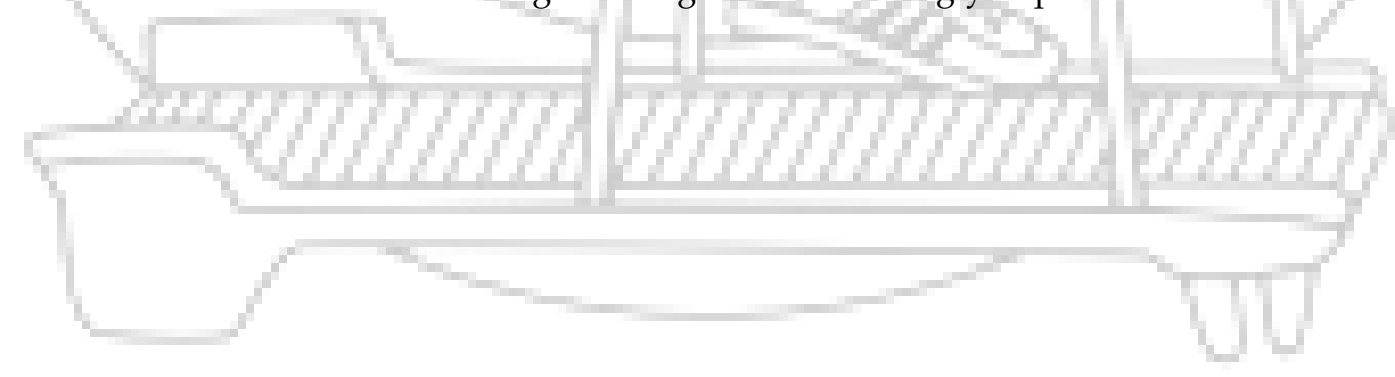

